\title{
Editorial
}

\section{Gynecological cancers, are we in the right direction to address this very important female health issue?}

\section{Dr. Syed A Aziz, Ph.D.}

Scientist, Health Canada,

Adjunct Professor, University of Ottawa, Canada

Corresponding Author Email: saziz@uottawa.ca

Focus of the matter is the female cancers are morphologically same but are clinically and etiologically different is almost every continent which makes it little difficult in managing and put a little more toll on clinicians. A cancer diagnosis is often linked to family medical history, lifestyle choices, or something in the environment. And while you can't control your family history or your whole environment, healthy lifestyle habits such as a good diet, regular physical activity, weight control, and quitting smoking if you're prone to lighting up are all within your control.

"Risk factors are individualized, but it's important to know that there are things you can do to lower your risk," understanding your risk factors and learning what you can do to modify them is key. Above stats are from one country only and based on that a rough estimate can be made for any country undertaking medical facilities especially for women. Irrespective of whatever the estimates are, an individual may or may not benefit, but talking with your doctor about your own risk factors based on your family history and lifestyle will certainly.

Knowing what you're up against can help you devise a plan for what screenings you may need to get (and when), what dietary changes could benefit you, and more - all personalized for you. "Modifying one thing might be helpful for one person but not necessarily helpful for someone else," in principal.

The concerned of focus on female cancer is due to the fact of breast cancer commonality globally and other gynecological cancers. According to WHO estimates it represents $10 \%$ of all cancers diagnosed worldwide and constituted $22 \%$ of all new cancers in 2000 in women making it the most common cancer in females. According to Research most female deaths by cancer is due to breast cancer, approximately 1 out of every 9 women are likely to suffer at any point in their lives and about $80 \%$ of women are diagnosed with invasive breast cancer over the age of 50, but if they were diagnosed then the survival rate approaches 90 . There is a trend of approx., 8-10\% increase in patient turnout each year. In 2017, an estimated $70-80 \%$ of new cases of invasive breast cancer are expected to be diagnosed in women in Pakistan., again this is from one country and to make the matter worse, the overall ignorance attitude towards the health of women by women in generally and especially by authorities in developing countries is of great concerned and deviate from the proper direction due to the lack of proper diagnostic facilities and treatment. It remains the most common malignancy in Pakistani females in both northern $(26.6 \%)$ and the southern parts $(20.8 \%)$ of the country. Karachi reports the highest incidence of breast cancer for any Asian population. Another concerned regarding breast cancer is the difference in mean age of the disease as the eastern women get breast cancer 10 years earlier than the western women.

All the above stats are to make a stronger case in terms of view of making awareness as common as possible, as knowing the factual position mean prevention and prevention is better than cure. Understanding the sign and symptoms of any disease let alone cancer will make things better for all. Whenever possible preforming the selfexamination is the best practice as individual knows the body well and also necessary for keeping a positive attitude toward one's own health. In a nut shell awareness and keeping a track of own health is the responsibility of an individual in order to fulfill the responsibility bestowed upon by the creator. 